\title{
Rehabilitación de las riberas de un sitio de la cuenca baja del río Matanza-Riachuelo
}

\author{
Laura Isabel de $\mathrm{Cabo}^{1}$, Ana Faggl ${ }^{2}$, Sebastián Miguel ${ }^{3}$ y Gabriel Basílico 2,4 \\ ${ }^{1}$ Museo Argentino de Ciencias Naturales "Bernardino Rivadavia" - Consejo Nacional de Investigaciones \\ Científicas y Técnicas (MACN-CONICET), CABA, Argentina. \\ ${ }^{2}$ Facultad de Ingeniería, Universidad de Flores (FI-UFLO), CABA, Argentina. \\ ${ }^{3}$ Laboratorio Bio-Ambiental de Diseño, Universidad de Flores, CABA, Argentina. \\ ${ }^{4}$ Laboratorio de Bioindicadores y Remediación, Universidad de Flores (LABIOC-UFLO), CABA, Argentina.
}

E-mail: Idecabo@macn.gov.ar

RESUMEN. La vegetación ribereña desempeña un rol importante en el control de fuentes no puntuales de contaminación y contribuye a la consiguiente mejora de la calidad del agua de ríos y arroyos. El río Matanza-Riachuelo ha sido clasificado como uno de los más contaminados del mundo, registrándose concentraciones elevadas de metales y otros contaminantes en la columna de agua y en los sedimentos de sus riberas. El objetivo de este trabajo consistió en realizar una caracterización física, química y florística y poner a prueba una intervención para la rehabilitación ecológica en un área piloto de la ribera del río Matanza-Riachuelo. Se determinaron cromo, cadmio, níquel, zinc, cobre y plomo en muestras de sedimentos ribereños y en muestras de las herbáceas Sagittaria montevidensis y Tradescantia fluminensis. Los suelos presentaron muy alto grado de contaminación, fundamentalmente debido a los elevados valores de cromo, cobre y zinc. Ambas herbáceas toleraron la variación del nivel del agua y acumularon metales, principalmente en sus estructuras subterráneas. Estas características indicaron que $S$. montevidensis y $T$. fluminensis podían ser utilizadas en intervenciones para la rehabilitación de riberas. La rehabilitación consistió en la plantación directa de 45 ejemplares de árboles y arbustos nativos con tolerancia al estrés ambiental (Erythrina crista-galli, Senna corymbosa, Solanum granulosum-leprosum, Allophylus edulis, Syagrus romanzoffiana, Salix humboldtiana, Lantana camara, Cestrum parqui y Sesbania punicea); en la línea de costa se instalaron 66 biorrollos para la implantación de las helófitas nativas: Hydrocotyle bonariensis, Tripogandra diuretica, T. fluminensis, Schoenoplectus californicus, S. montevidensis y Echinodorus grandiflorus. El monitoreo posterior demostró que los árboles y arbustos toleraron tanto los niveles de contaminación de los suelos como el prolongado periodo de inundación. La implantación de macrófitas S. californicus, S. montevidensis y E. grandiflorus mediante la técnica de biorrollos fue una alternativa eficaz pero los helófitos rastreros: H. bonariensis, T. fluminensis y $T$. diuretica resultaron afectados fundamentalmente por el depósito de basura flotante.

Palabras clave: Biorrollos, helófitas, metales pesados, sedimentos.

ABSTRACT. Riparian vegetation plays an important role in the control of non-point sources of pollution and the improvement of the water quality of rivers and streams. The Matanza-Riachuelo River has been classified as one of the most polluted in the world, according to high levels of metals and other pollutants in the water column and in the sediments of its banks. The objective of this work was to carry out a physical, chemical and floristic characterization and to test an intervention for ecological rehabilitation in a riparian pilot area of the Matanza-Riachuelo River. Chromium, cadmium, nickel, zinc, copper and lead were determined in sediment and samples of the herbaceous: Sagittaria montevidensis and Tradescantia fluminensis. Both herbaceous plants tolerate the variation of water level and accumulate metals, mainly in their underground structures. These characteristics indicate that $S$. montevidensis and $T$. fluminensis could be used in the rehabilitation of banks. The rehabilitation consisted in the direct planting of 45 specimens of native trees and shrubs tolerant 
de Cabo et al.

to environmental stress (Erythrina crista-galli, Senna corymbosa, Solanum granulosum-leprosum, Allophylus edulis, Syagrus romanzoffiana, Salix humboldtiana, Lantana camara, Cestrum parqui y Sesbania punicea), in the coastal line were installed 66 prevegetated rolls for the implantation of the native helophytes: Hydrocotyle bonariensis, Tripogandra diuretica, T. fluminensis, Schoenoplectus californicus, S. montevidensis y Echinodorus grandiflorus. The subsequent monitoring showed that the trees and shrubs tolerated both the levels of soil contamination and the prolonged period of flooding. The implantation of macrophytes (S. californicus, S. montevidensis y E. grandiflorus) by the technique of prevegetated rolls proved to be a good alternative, but the helophytes: $H$. bonariensis, $T$. fluminensis y $T$. diuretica were affected mainly by depositing floating debris.

Keywords: Heavy metals, helophytes, prevegetated rolls, sediments.

\section{INTRODUCCIÓN}

Las actividades humanas han provocado el deterioro de muchos ecosistemas acuáticos. La contaminación de los suelos de las áreas ribereñas es uno de los principales conflictos que surgen a la hora de plantear la rehabilitación de cuencas y la posterior recuperación del espacio para uso público. Estas zonas actúan como barreras físicas y químicas que atrapan nutrientes y contaminantes. Las interacciones que tienen lugar en la interfase agua-suelosedimento de las áreas ribereñas determinan y condicionan el nivel de disponibilidad de contaminantes para la biota. Por otra parte, las plantas que crecen en sitios contaminados constituyen herramientas que permiten evaluar distintos niveles de contaminación del suelo, y a la vez pueden ser aplicadas en técnicas de fitorremediación (Shah \& Nongkynrih, 2007). Las especies utilizadas para este propósito deben tolerar elevados niveles de contaminantes y ser capaces de acumularlos en distintos tejidos (Ali et al., 2013). Por otra parte, las especies que crecen en las riberas son además tolerantes a las fluctuaciones del nivel de agua. La vegetación ribereña desempeña un rol importante en el control de fuentes no puntuales de contaminación y en la consiguiente mejora de la calidad del agua de los cauces.

Las situaciones ambientales que tienen potencial de restauración son aquellas en las que el disturbio puede ser eliminado (Goodwin et al., 1997). En los casos de los ríos de llanura urbana y periurbana con una larga y compleja historia de degradación, el término rehabilitación resulta más apropiado para describir el enfoque de recuperación del estado ambiental ripario, debido a las inevitables limitaciones del entorno. Esta rehabilitación debería ser lograda con el mínimo aporte de energía posible, manipulando elementos del ecosistema y, preferiblemente, promoviendo procesos reversibles. A largo plazo, este enfoque resulta más eficiente que intentar controlar o reemplazar todas las funciones naturales por intervenciones humanas (Jungwirth et al., 2002). Sumado a esto, la mejora, en cuanto a la revaloración de los aspectos social, económico, estético y cultural, es de gran relevancia en los ambientes con fuerte influencia antrópica (Schanze et al., 2004).

El río Matanza-Riachuelo, es un río cuyas características hidrológicas no facilitan la evacuación de las cargas de contaminantes y ha sido clasificado como uno de los más contaminados del mundo (Magdaleno et al., 2014). La contaminación que afecta a este sistema es mixta: recibe efluentes líquidos y desechos tanto de origen industrial como doméstico, encontrándose entre estos últimos desechos cloacales y líquidos lixiviados de basurales que percolan a la napa freática y eventualmente descargan en el río. Existe una variedad de contaminantes presentes en los cuerpos de agua y sedimentos de la cuenca como ser hidrocarburos alifáticos y aromáticos, plaguicidas, bifenilospoliclorados (BPCs), compuestos orgánicos persistentes y metales pesados (plomo, cromo, mercurio, cadmio, zinc, cobre) (Casares y de Cabo, 2018). El río ha sido canalizado, propiciando tanto la 
pérdida de áreas ribereñas como de humedales en su cuenca. Además, las riberas han sido deforestadas y en algunas oportunidades reforestadas con especies exóticas. El efecto general de todas estas intervenciones antrópicas ha llevado a reducir drásticamente la riqueza biológica de la cuenca (Melignani, 2017). Por todo lo expuesto, y de acuerdo con la definición de Brown y Lugo (1994), la cuenca MatanzaRiachuelo es una cuenca altamente degradada. La rehabilitación de cuencas degradadas es una prioridad en el manejo de recursos en muchos países. En el marco de la actualización del Plan Integral de Saneamiento propuesto para la cuenca del río Matanza-Riachuelo (PISA) presentado en 2016 (http://www.acumar.gob. ar/wp-content/uploads/2016/12/PISA-2016. pdf), se han planeado acciones tendientes al ordenamiento territorial a partir de la recuperación de espacios públicos y la mejora en las condiciones habitacionales de la población. La recuperación de espacios, las nuevas vías de circulación, la limpieza de las márgenes y el espejo de agua, forman parte del trabajo orientado a poner en valor el camino de sirga de la cuenca (ACUMAR, 2019).

En el área ribereña de los ríos y arroyos de los alrededores de Buenos Aires la vegetación corresponde a las de las comunidades de matorral ribereño y ceibal (Cabrera, 1953). La vegetación de estos ambientes está dominada por arbustos y árboles de mediano porte: Erythrina crista-galli L., Sapium haematospermum Müll. Arg., Tessaria integrifolia Ruiz \& Pav., Salix humboldtiana Willd., Lantana camara L., Hibiscus striatus Cav., Phyllantus sellowianus (Klotzsch) Müll. Arg., Cephalanthus glabratus (Spreng.) K. Schum. y Sesbania punicea (Cav.) Benth.; gramíneas y otras herbáceas crecen cerca de la línea de costa: Sagittaria montevidensis Cham. \& Schltdl., Schoenoplectus californicus (C.A. Mey.) Soják, Alternanthera philoxeroides (Mart.) Griseb., Paspalum distichum L., Panicum pernambucense (Spreng.) Pilg., Echinodorus macrophyllus (Kunth) Micheli, Hydrocotyle ranunculoides L., $H$. bonariensis Lam., Polygonum punctatum Elliott, Pontederia cordata L., P. rotundifolia L., Senecio bonariensis Hook. \& Arn., Echinochloa helodes (Hack.) Parodi, Tradescantia fluminensis Vell. y en áreas remansadas es posible encontrar plantas flotantes como Pontederia crassipes Mart. Sin embargo, esta vegetación autóctona ha sido parcialmente reemplazada por vegetación exótica (Faggi y Breuste, 2014). Algunas de las especies mencionadas tienen la capacidad de tolerar, acumular y/o estabilizar contaminantes en sus tejidos. Mendoza et al. (2015) encontraron que Eleocharis montana (Kunth) Roem. \& Schult., Cyperus eragrostis Lam. y H. bonariensis podían concentrar metales pesados en sus raíces. Ejemplares de $S$. montevidensis colectados en las riberas del Riachuelo son capaces de tolerar elevados niveles de cromo, zinc y plomo y de acumularlos en sus raíces (Serafini et al., 2007). Arreghini et al. (2001) hallaron que $S$. californicus podía retener metales en sus rizomas. Pontederia crassipes y $P$. azurea Sw. mostraron gran tolerancia a altas concentraciones de cobre y cadmio en agua y en suelo, respectivamente, y podían acumularlos en raíces, siendo ambas especies adecuadas para la aplicación en fitorremediación de aguas y suelos contaminados (Melignani et al., 2015, 2019).

Una estrategia comúnmente adoptada para la rehabilitación de las cuencas hidrográficas es la implantación de vegetación ribereña. McKergow et al. (2003) hallaron que la presencia de vegetación riparia reducía 10 veces la exportación de sedimentos por erosión mientras que Yeakley et al. (2003) describen un aumento de cuatro veces en la concentración de nitratos en el agua subterránea en un área con remoción de la vegetación riparia. Los diversos procesos por los cuales la vegetación ribereña influye en la química del agua varían desde la absorción química directa y el estado fenológico de las plantas hasta influencias indirectas, como el suministro de detritos químicamente activos a los suelos y cauces, la modificación del movimiento del agua y la estabilización del suelo (Dosskey et al., 2010). La vegetación riparia actúa como estabilizadora de contaminantes, acumulándolos fundamentalmente en sus tejidos subterráneos, disminuyendo el ingreso de contaminantes por escorrentía, la erosión ribereña $y$, consecuentemente, mejorando la calidad del agua. La rehabilitación de ambientes 
acuáticos basada en la revegetación con especies nativas favorece el restablecimiento de fauna, especialmente aves e insectos, los cuales utilizan la cubierta vegetal como áreas para reproducirse, anidar, alimentarse y refugiarse. Asimismo, la presencia de áreas riparias vegetadas favorece la acumulación de sedimentos especialmente en el entramado de raíces, por la disminución de la velocidad del flujo, característica que incrementa el tamaño de los bancos de semillas (Dosskey et al., 2010). El proceso de rehabilitación debe comenzar por la selección de los sitios lo que requiere un estudio previo que permita evaluar los beneficios ecológicos y las posibilidades de éxito del plan en cada localización y definir en consecuencia sitios prioritarios para ser rehabilitados. Para ello es importante realizar una caracterización ambiental a través de indicadores de calidad de ribera (Basílico et al., 2015) y una posterior caracterización física, química y biológica del sitio seleccionado.

El objetivo de este trabajo consistió en realizar una caracterización física, química y florística en un área piloto de la ribera del río MatanzaRiachuelo y poner a prueba una propuesta de rehabilitación ecológica

\section{MATERIALES Y MÉTODOS}

Selección del sitio para llevar a cabo la rehabilitación ecológica de ribera

Durante febrero de 2015 se navegó el tramo Riachuelo del río Matanza-Riachuelo, aguas abajo del Puente La Noria hasta su desembocadura en el Río de la Plata (Figura 1). Desde el punto de vista urbano-ambiental se identificaron seis posibles zonas de intervención: Puente La Noria, desembocadura del arroyo Cildañez, Puente Alsina (Puente Uriburu o Puente Ezequiel Demonty), Villa 21-24 (aguas arriba del meandro de Brian), Puente Victorino de la Plaza y Puente del Ferrocarril General Roca (Figura 2). La selección de los sitios se realizó a partir del estado de la ribera y del impacto positivo para la población y la visibilidad que podría tener la futura intervención en cada

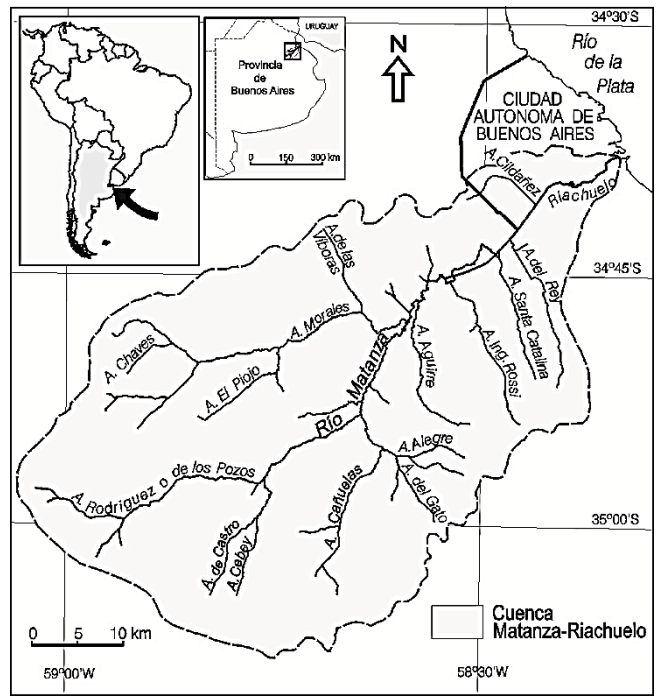

Figura 1. Mapa de la cuenca Matanza-Riachuelo. Figure 1. Map of the Matanza-Riachuelo basin.

una de las localizaciones. Se aplicó el Índice de Calidad de Riberas Pampeanas (ICRP) a cada uno de los sitios ya que tiene en cuenta la conectividad con ecosistemas adyacentes, los aportes laterales, tipo y uso de suelo en la ribera, grado de cobertura vegetal y calidad de esta, pendiente del terreno y naturalidad del canal fluvial (Basílico et al., 2015).

\section{Caracterización física, química y florística del sitio seleccionado}

A partir de la determinación de la calidad de riberas en los seis sitios del ítem anterior se seleccionó un área para realizar la intervención: Puente Alsina (Puente Uriburu o Ezequiel Demonty) donde se realizó la caracterización física y química de los suelos de ribera. Las muestras de suelos y sedimentos fueron tomadas a $10 \mathrm{~cm}$ de la superficie durante marzo de 2015. Se trazaron cuatro transectas perpendiculares al curso de agua, que incluyeron tres puntos cada una (Figura 3) pudiendo entonces definirse la Línea de costa, Centro (8,810,9 m de la línea de costa) y Alto (14,4-18,14 m de la línea de costa). Línea de costa incluyó las muestras S1; S4; S7 y S10, las de Centro fueron 

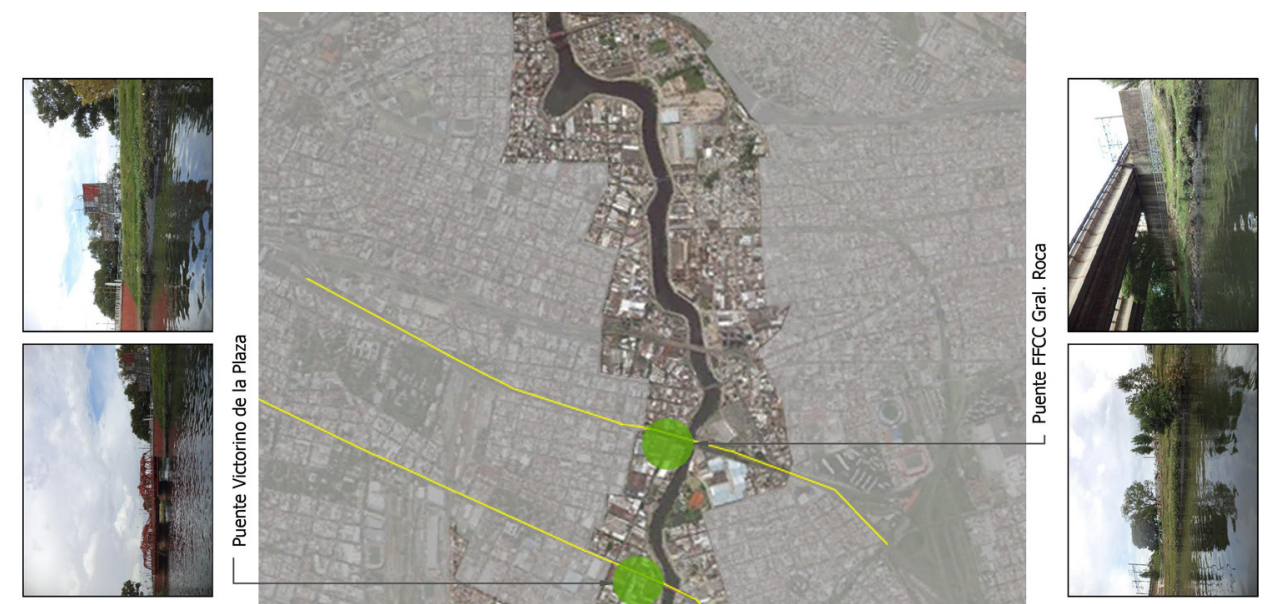

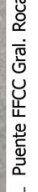
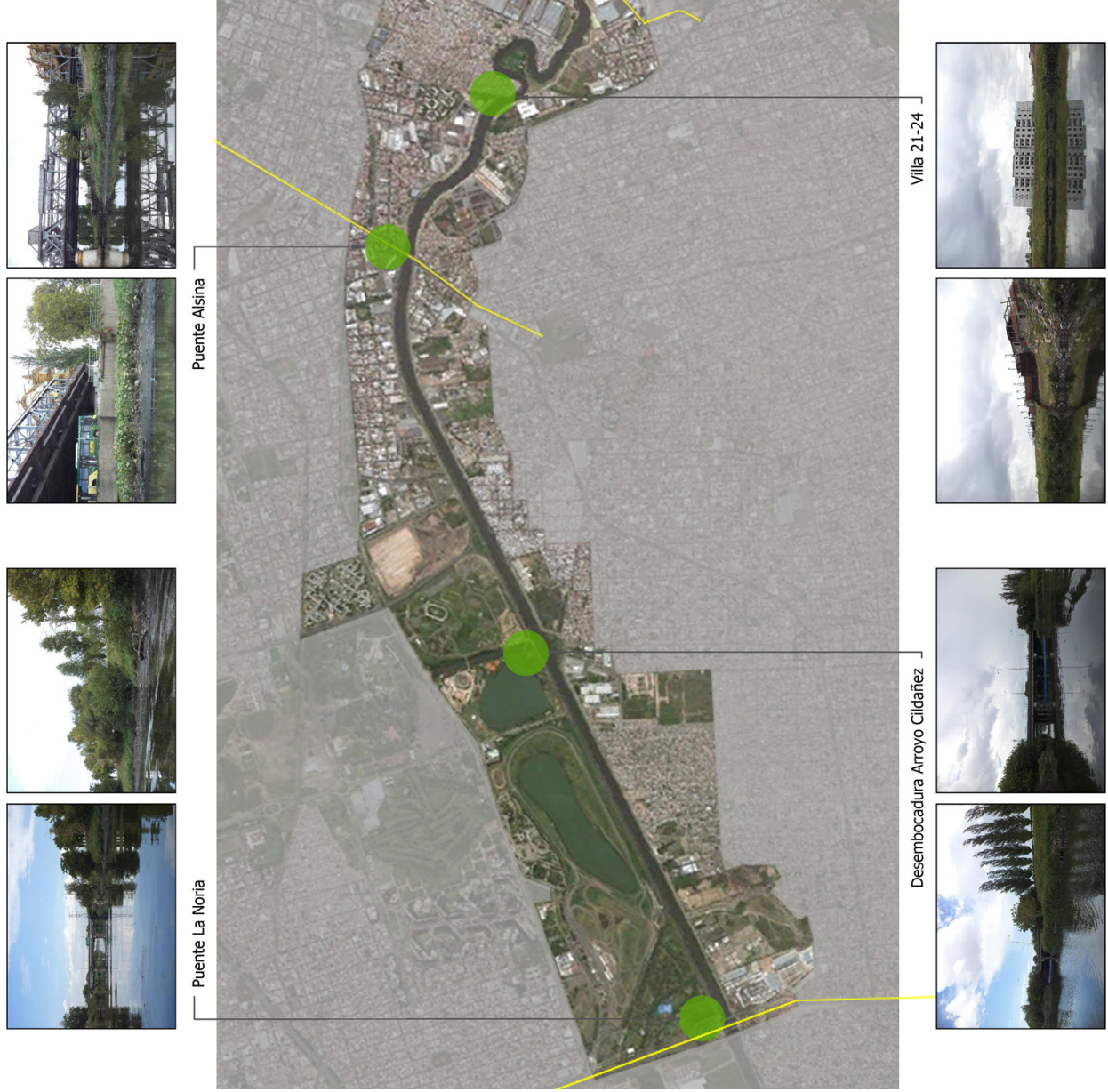

Figura 2. Ubicación de las seis posibles zonas de intervención: Puente La Noria, Desembocadura del arroyo Cildañez, Puente Alsina (Puente Uriburu o Puente Ezequiel Demonty), Villa 21-24 (aguas arriba del meandro de Brian), Puente Victorino de la Plaza y Puente del Ferrocarril General Roca.

Figure 2. Location of the six possible intervention areas: La Noria Bridge, Cildañez streammouth, Alsina Bridge (Uriburu Bridge or Ezequiel Demonty Bridge), Villa 21-24 (upstream of Brian's meander), Victorino de la Plaza Bridge and Bridge Railroad General Roca. 
S2; S5; S8 y S11 y en el caso de Alto S3; S6; S9 y $\mathrm{S12}$. Se determinaron las concentraciones de cromo, cadmio, níquel, zinc, cobre y plomo por espectrometría de absorción atómica (EAA) con digestión ácida previa de hidrocarburos totales (C8-C35) según TNRCC (2001). Se determinaron las variables edáficas: $\mathrm{pH}$ (relación suelo: agua $1: 2,5)$, conductividad eléctrica (CE), materia orgánica porcentual (\%MO) (Storer, 1984) y granulometría por tamizado y pipeteo (Day, 1965).

Se calculó un índice de contaminación ( $\left.D_{c}\right)$ (Kwon and Lee, 1998):

$$
\mathrm{D}_{\mathrm{c}}=\sum_{\mathrm{m}=1}^{\mathrm{n}}\left(\frac{\mathrm{C}_{\mathrm{m}}}{\mathrm{C}_{\mathrm{ref}}}\right)
$$

Siendo $C_{m}$ la concentración media de metal en el área y $C_{\text {ref }}$ la concentración de referencia de cada metal. Esta relación se denomina $C_{f}$. Para esta estimación se consideró como suelo de referencia un Natraquol Típico, utilizado por Mendoza et al. (2015) para un estudio de contaminación de suelos de la cuenca MatanzaRiachuelo.

Se identificaron todas las especies de plantas vasculares presentes en cada una de las 12 muestras (Figura 3), estimando visualmente su porcentaje de cobertura según el método fitosociológico de Braun-Blanquet (1979). Los ejemplares que no contaban con estructuras reproductivas o frutos se determinaron a nivel de género. Los ejemplares restantes que no pudieron ser identificados se descartaron del análisis (menos del 5\% de las muestras). La nomenclatura de las especies siguió a Zuloaga y Morrone (1996, 1999a, 1999b) y Zuloaga et al. (1994), con la actualización del Catálogo de las Plantas Vasculares del Cono Sur del Instituto de Botánica Darwinion (IBODA, 2019).

Además, se tomaron muestras de plantas adultas completas de dos especies de vegetación herbácea nativa que presentaban elevada cobertura en el tramo: $S$. montevidensis y $T$. fluminensis (parte aérea y subterránea) de tamaño similar y el suelo asociado. Para las determinaciones de metales pesados en las distintas partes de la planta, el material vegetal cosechado se secó hasta peso constante y se molió, realizando luego una digestión con $\mathrm{HNO}_{3}-\mathrm{HClO}_{4}$. Las concentraciones en todos los extractos fueron medidas por EAA con llama aire/acetileno. Se determinaron los contenidos totales de cromo, cadmio, níquel, zinc, cobre y plomo.

\section{Intervención realizada}

Durante los meses de marzo y abril 2015 se cultivaron las helófitas nativas: S. montevidensis, S. californicus, E. grandiflorus, H. bonariensis, $P$. cordata, T. fluminensis y Tripogandra diuretica (Mart.) Handlos en biorrollos con el objeto de que puedan desarrollar su sistema radicular (Figura 4). En abril de 2015 se realizó la intervención en un área de $200 \mathrm{~m}$ inmediatamente aguas

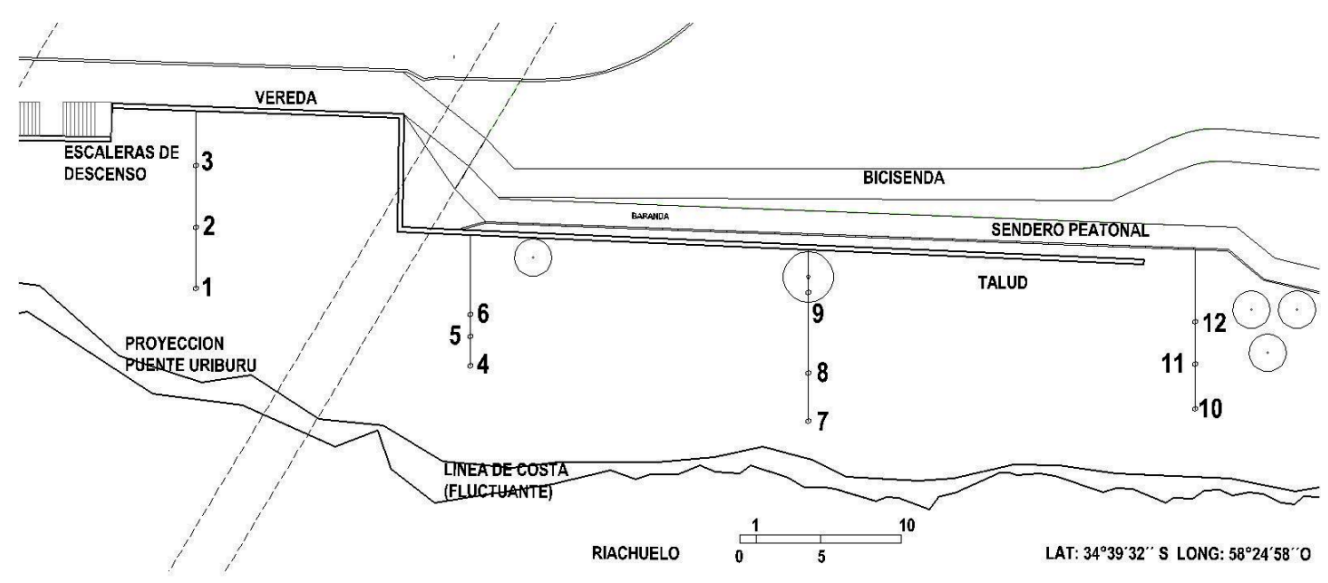

Figura 3. Esquema con los sitios de muestreo en la margen izquierda del Riachuelo, aguas abajo de Puente Uriburu (Puente Ezequiel Demonty).

Figure 3. Scheme with sampling sites on the leftbank of the Riachuelo, downstream Uriburu Bridge (Ezequiel Demonty Bridge). 


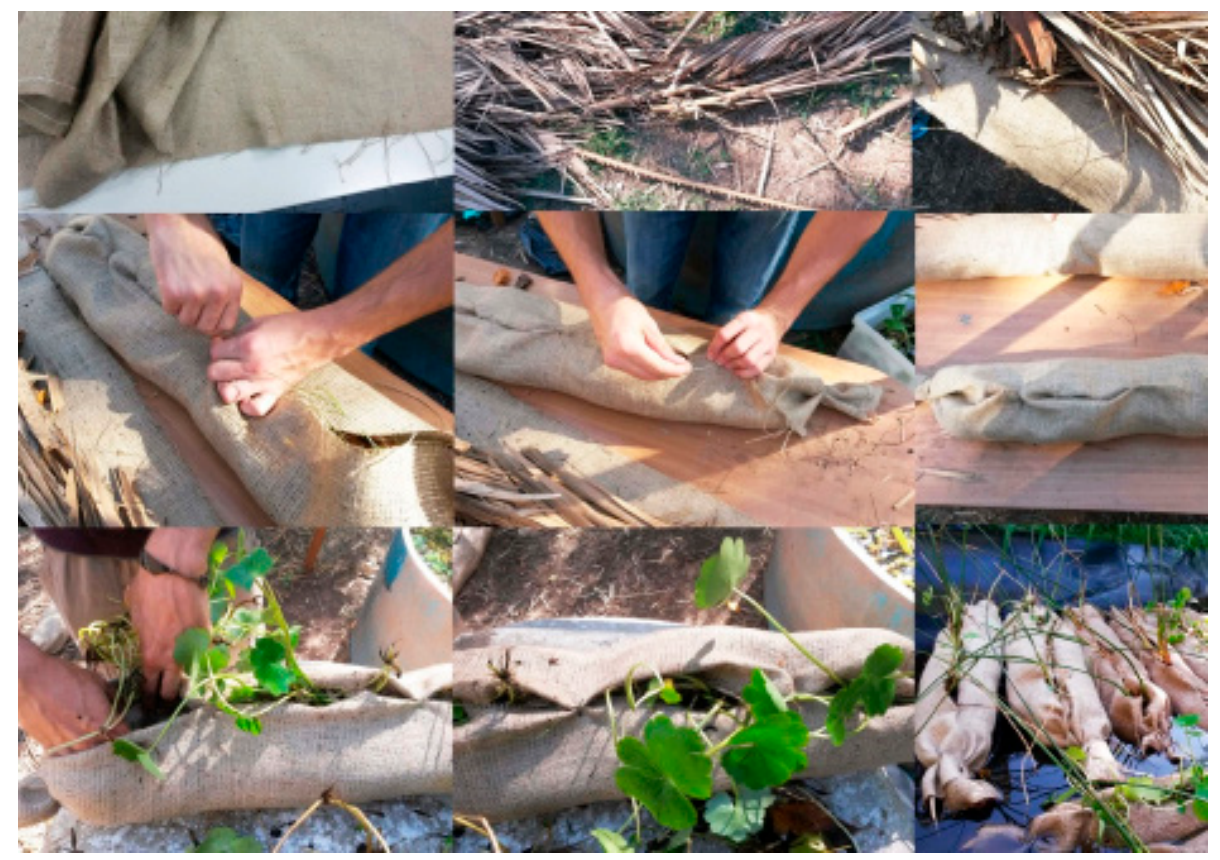

Figura 4. Secuencia del armado del biorrollos para implantación de helófitas nativas.

Figure 4. Sequence of the manufacture of prevegetated rolls for the implantation of the native helophytes.

abajo de Puente Alsina sobre la ribera Norte (Figura 5) y consistió en la plantación directa de 45 ejemplares de árboles y arbustos nativos de alturas superiores a 1 metro (E. crista-galli, Senna corymbosa (Lam.) H.S. Irwin \& Barneby, Solanum granulosum-leprosum Dunal, Allophylus edulis (A. St.-Hil., A. Juss. \& Cambess.) Hieron. ex Niederl., Syagrus romanzoffiana (Cham.) Glassman, $S$. humboldtiana, L. camara, Cestrum parqui L'Hér. y S. punicea). En los sectores de la línea de costa con menor cobertura, se aplicaron 66 biorrollos de $1 \mathrm{~m} \times 0,20 \mathrm{~m}$ en grupos de seis fijados al terreno con sogas para evitar los posibles desplazamientos por crecidas o bajantes del río. Se realizaron visitas mensuales al predio para realizar el seguimiento de la intervención realizada. En octubre 2015, se identificaron todas las especies de plantas vasculares presentes en cada una de las 12 muestras (Figura 3) para determinar la supervivencia de árboles y helófitas implantados, aplicando la metodología explicada previamente.

\section{RESULTADOS Y DISCUSIÓN}

La aplicación del Índice de Calidad de Riberas Pampeanas (ICRP) (Basílico et al., 2015) arrojó calidades de ribera de muy malas a intermedias para los seis sitios (Tabla 1).

El sitio seleccionado como área piloto fue un sector de 200 metros sobre la margen Norte, aguas abajo de Puente Alsina. La elección se basó en las siguientes características: mala calidad de ribera (Tabla 1), cercanía a áreas pobladas, pendientes poco pronunciadas, baja cubierta vegetal, acceso seguro al área, área incluida en el camino de sirga habilitado, ícono de la ciudad de Buenos Aires. Dicho sitio es un buen ejemplo de ribera que en un contexto urbano tendría potencial para la rehabilitación socioambiental (Guida Johnson, 2015). Bajo este concepto se entiende que es plausible realizar medidas cuyo objetivo sea crear áreas verdes que puedan ser usadas con fines de recreación, participación social y/o educación ambiental. 
de Cabo et al.

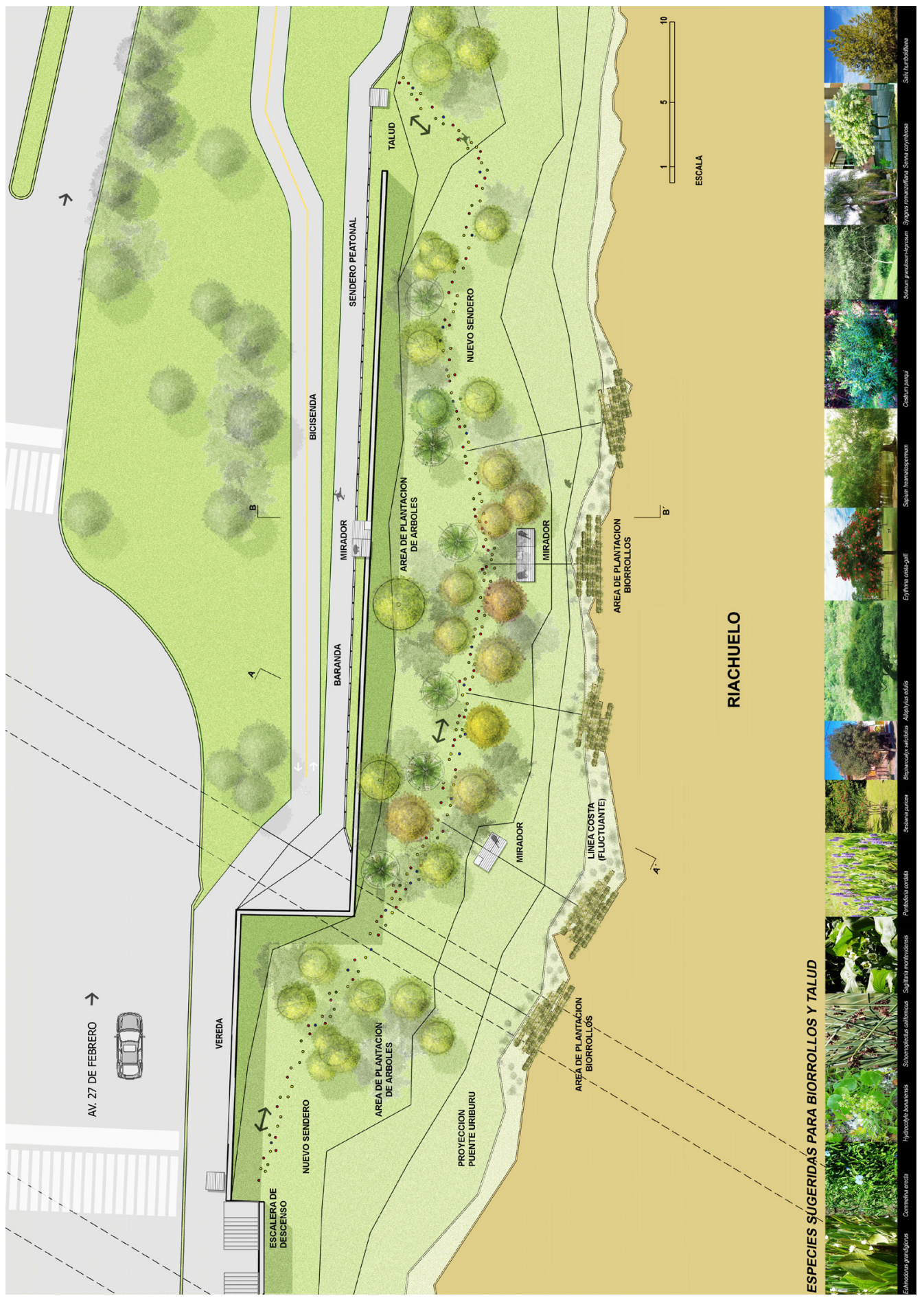

Figura 5. Propuesta de rehabilitación para la margen Norte del río Matanza-Riachuelo aguas abajo de Puente Alsina (Pte. Uriburu o Puente Ezequiel Demonty).

Figure 5. Rehabilitation proposal for the northbank of the Matanza-Riachuelo River downstream of Alsina Bridge (Pte. Uriburu or Ezequiel Demonty Bridge). 
Tabla 1. Valores del Índice de Calidad de Riberas Pampeanas (ICRP) y clasificación de la calidad de ribera según ICRP (C-ICRP) para los seis sitios de la Cuenca Baja del río Matanza-Riachuelo.

Table 1. Values of the Quality Index of the Pampas Riverbanks (ICRP) and classification of the quality of riverbank (C-ICRP) for the six sampling sites at the Lower Basin of the Matanza-Riachuelo River.

\begin{tabular}{lcc}
\hline Sitio & ICRP & C-ICRP \\
\hline Puente La Noria & 45 & Intermedia \\
Desembocadura del Ao. Cildañez & 34 & Mala \\
Puente Alsina & 34 & Mala \\
Villa 21-24 & 20 & Muy mala \\
Puente Victoriano de la Plaza & 30 & Mala \\
FFCC Gral. Roca cargas & 23 & Muy mala \\
\hline
\end{tabular}

\section{Caracterización fisicoquímica de los suelos del área de intervención}

Los suelos fueron neutros, lo cual asegura una buena disponibilidad de nutrientes para las plantas. Con el propósito de determinar el nivel de interacción entre el área riparia y el curso de agua, se midieron las principales variables a tres distancias desde la línea de costa. Los menores valores promedio de materia orgánica se midieron en la línea de costa y se incrementaron al alejarse de ésta. Los niveles de conductividad fueron bajos y se pueden clasificar como suelos no salinos (Tabla 2). Los niveles de metales medidos superaron los niveles guía propuestos para uso residencial por la legislación argentina, en el caso de cromo total y cobre (Tabla 3). También superaron los niveles propuestos por Francia, Canadá y Suecia (Tabla 3) en el caso de zinc, cromo total y cobre, para el mismo uso. Al considerar los niveles por encima de los cuales se espera que sea más probable que ocurran efectos biológicos adversos (PEL: por sus siglas en inglés, Probable Effect Level), el cromo total en las tres distancias a la línea de costa, el plomo en la distancia intermedia y el zinc en distancias intermedia y alta los superaron. Las determinaciones de hidrocarburos totales sólo superaron los niveles de detección en algunos sitios de la línea de costa. Las tendencias observadas en las concentraciones respecto a la distancia de la línea de costa revelan que el cromo total disminuye al alejarse de la costa mientras que el resto de los metales presentan las mayores concentraciones en una posición intermedia (Centro).

Los suelos analizados son hídricos ya que

Tabla 2. Principales variables edáficas y rango de las concentraciones de metales pesados e hidrocarburos totales en muestras de suelos. El asterisco $\left({ }^{*}\right)$ indica los valores que superaron los niveles guía para suelos de uso residencial, contemplados por la legislación argentina (Decreto Reglamentario 831/93, Ley 24.051). ND: dato no disponible.

Table 2. Main edaphic variables and range of concentrations of heavy metals and total hydrocarbons in soil samples. The asterisk $\left({ }^{*}\right)$ indicates the values that exceeded the guide levels for residential use, contemplated by Argentine legislation (Regulatory Decree 831/93, Law 24.051). ND: data not available

\begin{tabular}{lccc}
\hline Variable & Línea de Costa & Centro & Alto \\
\hline $\mathrm{pH}$ & $7,16 \pm 0,12$ & $6,93 \pm 0,06$ & $6,93 \pm 0,03$ \\
$\mathrm{CE}(\mu \mathrm{S} / \mathrm{cm})$ & $543 \pm 32$ & $610 \pm 125$ & $665 \pm 186$ \\
$\% \mathrm{MO}(\mathrm{i})$ & $8,12 \pm 1,33$ & $13,02 \pm 2,74$ & $11,52 \pm 0,49$ \\
Cromo total $(\mathrm{mg} / \mathrm{kg})$ & $146-325^{*}$ & $34-142$ & $30-116$ \\
Cadmio $(\mathrm{mg} / \mathrm{kg})$ & $\mathrm{ND}$ & $\mathrm{ND}-0,8$ & $\mathrm{ND}-0,4$ \\
Zinc $(\mathrm{mg} / \mathrm{kg})$ & $233-244$ & $330-417$ & $272-384$ \\
Plomo $(\mathrm{mg} / \mathrm{kg})$ & $35-42$ & $40-135$ & $37-62$ \\
Niquel $(\mathrm{mg} / \mathrm{kg})$ & $9-12$ & $10-13$ & $8-13$ \\
Cobre $(\mathrm{mg} / \mathrm{kg})$ & $68-83$ & $66-102 *$ & $53-108$ \\
$\begin{array}{l}\text { Hidrocarburos Totales } \\
\text { (C8 - C35) }(\mathrm{mg} / \mathrm{kg})\end{array}$ & $\mathrm{ND}-204$ & $\mathrm{ND}$ & $\mathrm{ND}$ \\
\hline
\end{tabular}


Tabla 3. Niveles guía de la concentración de metales pesados para suelos de uso residencial. * Cr total. (1) Decreto Reglamentario 831/93, Ley 24.051; (2) Canadian Soil Quality Guidelines for the Protection of Environmental and Human Health; (3) Provoost et al., 2006; (4) Swedish Environmental Protection Agency y (5) Nivel de Efecto Probable (PEL, por sus siglas en inglés, Probable Effect Level) del Canadian Council of Minister of the Environment. Table 3. Guidance levels of heavy metal concentration in soil for residential use. * Total Cr. (1) Regulatory Decree 831/93, Law 24.051; (2) Canadian Soil Quality Guidelines for the Protection of Environmental and Human Health; (3) Provoost et al., 2006; (4) Swedish Environmental Protection Agency and (5) Probable Effect Level (PEL) of the Canadian Council of Minister of the Environment.

\begin{tabular}{lcccccc}
\hline Uso residencial & Cadmio & $\begin{array}{c}\text { Cromo III } \\
\mathrm{mg} / \mathrm{kg} \text { peso seco }\end{array}$ & $\begin{array}{c}\text { Cobre } \\
\text { perinc }\end{array}$ & Plomo & Níquel & Zinc \\
\hline Argentina (1) & 5 & $250^{*}$ & 100 & 500 & 100 & 500 \\
Canadá (2) & 10 & 64 & 63 & 140 & 50 & 200 \\
Francia (3) & 20 & $130^{*}$ & 190 & 400 & 140 & 9000 \\
Suecia (4) & 0,4 & 120 & 100 & 80 & 35 & 350 \\
PEL (5) & 3,5 & $90^{*}$ & 197 & 91 & - & 315 \\
\hline
\end{tabular}

permanecen bajo agua la mayor parte del tiempo ya sea encharcados, inundados o saturados en agua (Vasilas et al., 2010). En consecuencia, se trata de suelos anaeróbicos donde tienen lugar procesos de reducción. Presentan sulfuros, producto de la reducción de sulfatos en un ambiente anóxico, lo cual otorga una coloración negruzca y olor penetrante.

El valor de Dc obtenido para el área de estudio (Tabla 4) reveló un muy alto grado de contaminación, fundamentalmente debido a los elevados valores de cromo ( $C f>6)$, cobre y zinc (Cf entre 3-6).

Los suelos estudiados resultaron ser mayoritariamente fango-arenoso, con abundancia de limo $(34,4 \%)$ y arcilla $(32,5 \%)$ (Tabla 5). Estos suelos con abundancia de fracciones finas (limos y arcillas) presentan mal drenaje y se producen fenómenos de interacción con los metales, fundamentalmente procesos de intercambio iónico y de adsorción superficial.

Las características edáficas, la presencia de sulfuros y la abundancia de materia orgánica favorecen los procesos de adsorción de los metales a la matriz del suelo, disminuyendo su disponibilidad para las plantas y el riesgo de movilidad de los mismos hacia otros compartimentos del sistema.

\section{Caracterización florística}

La vegetación estuvo representada por hierbas nativas como S. montevidensis, E. helodes, P. punctatum, Cyperus pohlii (Nees) Steud. y $T$. fluminensis, y cosmopolitas (Parietaria debilis G. Forst.) ( $70 \%$ de cobertura) y exóticas (30\% de cobertura) entre las que se contaban Cynodon dactylon (L.) Pers., Conyza bonariensis (L.) Cronquist, Sorghum halepense (L.) Pers., Digitaria sanguinalis (L.) Scop., Musa paradisiaca L. (banano). Se observó una enredadera típica de regiones cálidas (Ipomea cairica (L.) Sweet), arbustos nativos como Manihot grahamii Hook.

Tabla 4. Valores de factor de contaminación (Cf) y grado de contaminación (Dc) por metales en los suelos estudiados. Siendo $\mathrm{Cf}<1$ baja contaminación; Cf 1-3 moderada contaminación; Cf 3-6 alta contaminación, y Cf>6 muy alta contaminación. $\mathrm{Dc}<6$ bajo grado de contaminación; Dc 6-12 moderado grado de contaminación, y Dc 12-24 muy alto grado de contaminación, según Kwon and Lee (1998).

Table 4. Values of contamination factor (Cf) and degree of contamination (Dc) bymetals in the studied soils, being $C f<1$ low pollution; Cf 1-3 moderate pollution; $C f$ 3-6 high pollution, and $C f>6$ very high pollution. $D c<6$ low degree of contamination; Dc 6-12 moderate degree of contamination, and Dc 12-24 very high degree of contamination, according to Kwon and Lee (1998).

\begin{tabular}{lccc} 
Metal & $\mathrm{C}_{\mathrm{m}}(\mathrm{mg} / \mathrm{kg})$ & $\mathrm{C}_{\mathrm{ref}}(\mathrm{mg} / \mathrm{kg})$ & $\mathrm{C}_{\mathrm{f}}$ \\
\hline Cromo & 122 & 19 & 6,3 \\
Zinc & 318 & 74 & 4,3 \\
Plomo & 51 & 31 & 1,7 \\
Cobre & 82 & 23 & 3,5 \\
\hline
\end{tabular}


Tabla 5. Granulometría de las muestras de suelo a tres distancias de la línea de costa.

Table 5. Granulometry of soil samples at three distances from the coast line.

\begin{tabular}{cccc}
\hline $\begin{array}{c}\text { Fracción } \\
(\%)\end{array}$ & $\begin{array}{c}\text { Línea de } \\
\text { Costa }\end{array}$ & Centro & Alto \\
\hline Grava & $0,2 \pm 0,1$ & $0,7 \pm 1,4$ & $1,6 \pm 3,0$ \\
Arena & $37,3 \pm 10,1$ & $24,9 \pm 3,6$ & $34,6 \pm 12,9$ \\
Limo & $34,4 \pm 7,0$ & $36,7 \pm 2,2$ & $32,1 \pm 7,0$ \\
Arcilla & $28,2 \pm 3,3$ & $37,7 \pm 4,0$ & $31,7 \pm 6,1$ \\
\hline
\end{tabular}

(falso cafeto) y exóticos como Ricinus communis L. (ricino). La cobertura de los árboles alcanzaba el 40\%, la gran mayoría exóticos, representados por Morus sp. (mora), Casuarina cunninghamiana Miq. (casuarina), Platanus acerifolia Willd. (plátano) y la nativa Blepharocalyx salicifolius (Kunth) O. Berg (anacahuita).

\section{Contaminantes en la vegetación estudiada}

Las concentraciones de metales pesados medidos en $S$. montevidensis revelan que esta planta es capaz de absorber y acumular cromo, plomo, zinc y cobre en estructuras subterráneas, sin translocar a las hojas por encima de los valores definidos para plantas terrestres de sitios no contaminados (Nagajyoti et al., 2010), a excepción del cromo que superó los niveles de referencia para estructuras aéreas (Tabla 6). La especie $T$. fluminensis absorbió cromo, zinc, plomo, níquel y cobre, los que quedaron acumulados en la estructura subterránea y hojas. Se detectaron en hojas niveles de cromo, cobre y zinc (Tabla 7) superiores a los valores de referencia para plantas terrestres de áreas no contaminadas (Tabla 6).

Con el objeto de evaluar la capacidad de absorber y acumular metales en las raíces de $S$. montevidensis y $T$. fluminensis, se calcularon factores de bioconcentración: $\mathrm{FBC}=$ concentración del metal en raíz/concentración de metal en suelo (según Mendoza et al., 2015). Los FBC calculados para cada metal fueron mayores a 1 , en el caso de $T$. fluminensis, particularmente en el caso de cromo $(F B C=6,5)$. Los resultados revelan mayor bioconcentración de metales en las raíces de $T$. fluminensis respecto a $S$. montevidensis (Tablas 6 y 7 ).

A fin de evaluar la capacidad de S. montevidensis y $T$. fluminensis para el transporte de metales hacia las estructuras verdes (hojas), se calcularon los factores de translocación: $\mathrm{FT}=$ concentración del metal en hoja/concentración de metal en raíz (según Mendoza et al., 2015) (Tablas 6 y 7). Los factores de translocación (FT) fueron inferiores a 1 en todos los casos, revelando un mínimo transporte de metales hacia las estructuras verdes de las plantas, lo que representa una estrategia de tolerancia, al evitar la llegada del tóxico a las estructuras fotosintéticas. Además, esta estrategia disminuye el riesgo que esos metales ingresen a la trama trófica de herbívoros.

Seis meses después de la intervención (octubre 2015), se pudo constatar que todos los árboles

Tabla 6. Concentraciones medias de metales pesados en suelos y en Sagittaria montevidensis (hoja y raíz). Factores de bioconcentración( $\mathrm{FBC}=$ concentración del metal en raíz/concentración demetalensuelo)yFactores detranslocación (FT = concentración del metal en hoja/concentración de metal en raíz).

Table 6. Average concentrations of heavy metals in soils and in Sagittaria montevidensis (leaf and root). Bioconcentration factors $(B C F=$ root metal concentration $/$ soil metal concentration) and Translocation factors (TF = leaf metal concentration / root metal concentration).

\begin{tabular}{lcccccc}
\hline Metal & Suelo & $\begin{array}{c}\text { Sontevidensis } \\
\text { (raíz) }\end{array}$ & $\begin{array}{c}\text { S. montevidensis } \\
\text { (hoja) }\end{array}$ & $\begin{array}{c}\text { Valor de } \\
\text { referencia } \\
\star\end{array}$ & FBC & FT \\
\hline Cromo total & 34,1 & $16,1 \pm 2,62$ & $2,5 \pm 0,8$ & $0,2-1$ & 0,472 & 0,155 \\
Zinc $(\mathrm{mg} / \mathrm{kg})$ & 417 & $90,0 \pm 11,7$ & $7,1 \pm 2,4$ & $8-100$ & 0,216 & 0,079 \\
Plomo $(\mathrm{mg} / \mathrm{kg})$ & 135 & $10,1 \pm 5,4$ & ND & $1-13$ & 0,075 & - \\
Níquel $(\mathrm{mg} / \mathrm{kg})$ & 12,4 & $\mathrm{ND}$ & $\mathrm{ND}$ & 1 & - & - \\
Cobre $(\mathrm{mg} / \mathrm{kg})$ & 66,1 & $18,0 \pm 3,3$ & $2,5 \pm 1,0$ & $4-15$ & 0,272 & 0,134 \\
\hline
\end{tabular}

*Concentración en parte aérea en plantas terrestres de áreas no contaminadas ( $\mathrm{mg} / \mathrm{kg}$ peso seco) según Nagajyoti et al. (2010). 
Tabla 7. Concentraciones de metales pesados en suelos y en Tradescantia fluminensis (hoja y raíz). Factores de bioconcentración ( $\mathrm{FBC}=$ concentración del metal en raíz/concentración de metal en suelo) y Factores de translocación ( $\mathrm{FT}=$ concentración del metal en hoja/concentración de metal en raíz).

Table 7. Average concentrations of heavy metals in soils and in Tradescantia fluminensis (leaf and root). Bioconcentration factors $(B C F=$ root metal concentration / soil metal concentration) and Translocation factors (TF = leaf metal concentration / root metal concentration).

\begin{tabular}{|c|c|c|c|c|c|}
\hline Metal & Suelo & T. fluminensis (raíz) & T. fluminensis (hoja) & $F B C$ & $F T$ \\
\hline Cromo total (mg/kg) & 29,7 & $192 \pm 26$ & $54 \pm 21$ & 6,5 & 0,3 \\
\hline Zinc (mg/kg) & 272 & $280 \pm 13$ & $111 \pm 10$ & 1,0 & 0,4 \\
\hline Plomo(mg/kg) & 36,7 & $41 \pm 28$ & $11 \pm 3$ & 1,1 & 0,3 \\
\hline Níquel (mg/kg) & 8,2 & $8 \pm 2$ & ND & 1,0 & - \\
\hline Cobre & 52,6 & $74 \pm 12$ & $24 \pm 1$ & 1,4 & 0,3 \\
\hline
\end{tabular}

y arbustos implantados toleraron tanto los niveles de contaminación de los suelos como el prolongado periodo de inundación (3 meses) producto de las elevadas precipitaciones durante los meses julio a octubre 2015 superiores a las normales del periodo 19612000. En las muestras cercanas a la línea de costa: 4, 7 y 10 (Figura 3) no se detectó presencia de los helófitos rastreros implantados a través de biorrollos $(H$. bonariensis, $T$. fluminensis y $T$. diuretica) afectados fundamentalmente por el depósito de basura flotante. En cambio, $S$. montevidensis, S. californicus y E. grandiflorus también implantados a través de biorrollos, presentaron una supervivencia superior al $70 \%$. Para poder replicar esta experiencia en otras riberas deterioradas se requiere tener en cuenta las características de las mismas. Se pueden colocar biorrollos en la línea de costa, para consolidarla y evitar erosión, y crear un matorral bajo, en el talud superior utilizando arbolitos bajos (S. granulosum-leprosum, S. corymbosa). En la parte alta se podrían plantar algunos árboles aislados del bosque de albardón (Celtis tala Gillies ex Planch., Sambucus australis Cham. \& Schltdl., Schinus longifolius (Lindl.) Speg.). En riberas con pendiente muy pronunciada es importante mantener la cobertura existente para evitar el desmoronamiento del talud y el lavado del suelo hacia el río.

\section{CONCLUSIONES}

Los niveles de metales determinados en los suelos y sedimentos de las riberas del tramo estudiado del Riachuelo ponen de manifiesto un alto grado de contaminación, tanto por los elevados valores de cromo, en primer lugar, como de cobre y zinc, en segundo lugar. Las dos especies herbáceas estudiadas tienen la capacidad de tolerar la condición ambiental y acumular metales, fundamentalmente en sus estructuras subterráneas. El mantenimiento de estas especies en las riberas evitando su corte innecesario, limita la erosión y permite la estabilización de los metales en las estructuras subterráneas disminuyendo el riesgo que dichos contaminantes lleguen al curso de agua. Además, pueden ser implantadas en intervenciones para la rehabilitación de las riberas de la Cuenca del Matanza-Riachuelo y otras de características semejantes. Por otro lado, la implantación de helófitas con la técnica de biorrollos en las orillas demostró ser una buena alternativa de revegetación ya que los biorrollos otorgaron sostén y protección de las raíces. Sin embargo, la presencia de basura flotante limitó el crecimiento de la vegetación de la línea de costa. Por lo tanto se propone la implantación de una banda de herbáceas de gran porte (S. californicus, E. grandiflorus y $S$. montevidensis) a través de biorrollos en la línea de costa para limitar el ingreso y deposición de basura flotante y a continuación, implantar una línea de helófitos rastreros ( $H$. bonariensis, $T$. fluminensis y $T$. diuretica) mediante la técnica de biorrollos. 


\section{AGRADECIMIENTOS}

Este trabajo se desarrolló en el marco de un Convenio de Colaboración entre la Agencia de Protección Ambiental del GCBA y la Universidad de Flores: "Propuesta de restauración de la ribera Norte de la Cuenca Baja del Río MatanzaRiachuelo (2015)".

\section{REFERENCIAS}

ACUMAR. (2019). Plan Integral de Saneamiento Ambiental. Autoridad de Cuenca MatanzaRiachuelo. http://www.acumar.gob.ar/planintegral/.

Ali, H., Khan, E. \& Sajad, M. A. (2013). Phytoremediation of heavy metals - concepts and applications. Chemosphere, 91:869-881.

Arreghini, S., de Cabo, L., de lorio, A. F., Rendina, A. \& Bonetto, C. (2001). Effects of zinc on the growth of bulrush (Schoenoplectus californicus) and its distribution between different sediment fractions. Bulletin of Environmental Contamination and Toxicology, 67(2): 264-270.

Basílico, G. O., de Cabo, L. y Faggi, A. (2015). Adaptación de índices de calidad de agua y de riberas para la evaluación ambiental en dos arroyos de la llanura pampeana. Revista del Museo Argentino de Ciencias Naturales, nueva serie, 17(2): 119-134.

Braun-Blanquet, J. F. (1979). Fitosociología. Bases para el estudio de las comunidades vegetales. Madrid: Blume Ediciones.

Brown, S. \& Lugo, A. (1994). Rehabilitation of tropical lands: A key to sustaining development. Restoration Ecology, 2 (2): 97-111

Cabrera, A. L. (1953). Manual de la flora de los alrededores de Buenos Aires. Buenos Aires: ACME.

Casares, M. V y de Cabo, L. (2018). Análisis de tendencias de variables indicadoras de calidad de agua para el Riachuelo (Cuenca MatanzaRiachuelo, Argentina). Revista Internacional de Contaminación Ambiental, 34 (4): 651-657.
Day, P. R. (1965). Particle fractionation and particle size analysis. Methods of soil analysis. Part 1. Agronomy, 9: 545-566.

Burkart, A. E. (Dir.). (1974). Flora ilustrada de Entre Ríos (Argentina). Tomo 6 Parte VI: Dicotiledóneas Metaclamídeas (Gamopétalas). Rubiales, Cucurbitales, Campanulales. Buenos Aires: Instituto Nacional de Tecnología Agropecuaria.

Dosskey, M. G., Vidon, P., Gurwick, N. P., Allan, C. J., Duval, T. P. \& Lowrance, R. (2010). The role of riparian vegetation in protecting and improving chemical water quality in streams. Journal of the American Water Resources Association, 46 (2): 1-18.

Faggi, A. y Breuste, J. (2014). La cuenca MatanzaRiachuelo: Una mirada ambiental para recuperar sus riberas. Buenos Aires: Universidad de Flores.

Goodwin, C. N., Hawkins, C. P. \& Kershner, J. L. (1997). Riparian restoration in the western United States: Overview and perspective. Restoration Ecology, 5: 4-14.

Guida Johnson, B. (2015). Rehabilitación de ambientes degradados en la cuenca MatanzaRiachuelo: Enfoque a múltiples escalas. Universidad Nacional de General Sarmiento.

IBODA. (2019). Catálogo de las plantas vasculares del Cono Sur. Instituto de Botánica Darwinion. http://www2.darwin.edu.ar/Proyectos/ FloraArgentina/BuscarEspecies.asp.

Jungwirth, M., Muhar, S. \& Schmutz, S. (2002). Reestablishing and assessing ecological integrity in riverine landscapes. Freshwater Biology, 47(4): 867-887.

Kwon, Y. T. \& Lee, C. W. (1998). Application of multiple ecological risk indices for the evaluation of heavy metal contamination in a coastal dredging area. Science of the Total Environment, 214: 203-210.

Magdaleno, A., de Cabo, L., Arreghini, S. \& Salinas, C. (2014). Assessment of heavy metal contamination and water quality in an urban river from Argentina. Brazilian Journal of Aquatic Science and Technology, 18(1): 113-120. 
McKergow, L. A., Weaver, D. M., Prosser, I. P., Grayson, R. B. \& Reed, A. E. G. (2003). Before and after riparian management: sediment and nutrient exports from a small agricultural catchment, Western Australia. Journal of Hydrology, 270: 253-272.

Melignani, E. (2017). Pautas para la remediación y recuperación de áreas sujetas a contaminación mixta de cuencas urbanas y periurbanas de Ilanura. (Tesis Doctoral, Facultad de Ciencias Exactas y Naturales, UBA).

Melignani, E., de Cabo, L. I. \& Faggi, A. M. (2015). Copper uptake by Eichhornia crassipes exposed at high level concentrations. Environmental Science and Pollution Research, 22(11): 83078315.

Melignani, E; Faggi, A \& de Cabo, L. (2019). Growth, accumulation and uptake of Eichhornia crassipes exposed to high cadmium concentrations. Environmental Science and Pollution Research, 26(22): 22826-22834.

Mendoza, R., García. I, de Cabo, L., Weigandt, C. \& de Iorio, A. F. (2015). The interaction of heavy metals and nutrients present in soil and native plants with arbuscular mycorrhizae on the riverside in the Matanza-Riachuelo River Basin (Argentina). Science of the Total Environment, 505: 555-564.

Nagajyoti, P. C., Lee, K. D. \& Sreekanth, T. V. M. (2010). Heavy metals, occurrence and toxicity for plants: A review. Environmental Chemistry Letters, 8(3): 199-216.

Provoost, J., Cornelis, C. \& Swartjes, F. (2006). Comparison of soil clean-up standards for trace elements between countries: Why do they differ? Journal of Soils and Sediments, 6 (3): 173-181.

Schanze, J., Olfert, A., Tourbier, J. T., Gersdorf, I. \& Schwager, T. (2004). Existing urban river rehabilitation schemes. WP2 Final Report.Urban River Basin Enhancement Methods. RUBEM EU FP5 Project.

Serafini, R. J. M., Arreghini, S. \& de Iorio, A. F. (2007). Could Sagittaria montevidensis be used in phytoremediation strategies? In: Scarpati O. \& Jones J. A. A. (Eds.). Environmental Change and Rational Water Use (pp. 195-202). Buenos Aires: Orientación Gráfica Editora.

Shah, K. \& Nongkynrih, J. M. (2007). Metal hyperaccumulation and bioremediation. Biologia Plantarum, 51:618-634.

Storer, D. A. (1984). A simple high sample volume, ashing, procedure for determining soil organic matter. Communications in Soil Science and Plant Analysis, 15 (7): 759-772.

Vasilas, L. M., Hurt, G. W. \& Noble, C. V. (2010). Field indicators of hydric soils in the United States. United States Department of Agriculture, Natural Resources Conservation Service, in cooperation with the National Technical Committee for Hydric Soils, Washington, DC.

Yeakley, J. A., Coleman, D. C., Haines, B. L., Kloeppel, B. D., Meyer, J. L., Swank, W. T., Argo, B. W., Deal, J. M. \& Taylor, S. F. (2003). Hillslope nutrient dynamics following upland riparian vegetation disturbance. Ecosystems, 6: 154-167.

Zuloaga, F. O. y Morrone, O. (1996). Catálogo de las plantas vasculares de la República Argentina. I. Pteridophyta, Gymnospermae y Angiospermae (Monocotyledoneae). Monographs in Systematic Botany from the Missouri Botanical Garden, 60: 1-323.

Zuloaga, F. O. y Morrone, O. (1999a). Catálogo de las plantas vasculares de la República Argentina. II. Acanthaceae-Euphorbiaceae (Dicotyledoneae). Monographs in Systematic Botany from the Missouri Botanical Garden, 74: $1-621$

Zuloaga, F. O. y Morrone, O. (1999b). Catálogo de las plantas vasculares de la República Argentina. II. Fabaceae-Zygophyllaceae (Dicotyledoneae). Monographs in Systematic Botany from the Missouri Botanical Garden, 74: 1-1269.

Zuloaga, F. O., Nicora, E. G., Rúgolo de Agrasar, Z. E., Morrone, O., Pensiero, J. y Cialdella, A. M. (1994). Catálogo de la familia Poaceae en la República Argentina. Monographs in Systematic Botany from the Missouri Botanical Garden, 47: 1-178. 